\title{
Measuring Resident Well-Being: Impostorism and Burnout Syndrome in Residency
}

\author{
Jenny Legassie, BSc (Hon), MD', Elaine M. Zibrowski, BSc (Spec), MSc, MSc ${ }^{2}$, \\ and Mark A. Goldszmidt, MD, MHPE, FRCPC ${ }^{1,2}$ \\ 'Division of Internal Medicine, Department of Medicine, London Health Sciences Centre, London, ON, Canada; ${ }^{2}$ Group for the Advocacy \& \\ Advancement of Medical/Dental Education Scholarship (GAMES), Schulich School of Medicine \& Dentistry, University of Western Ontario, \\ London, ON, Canada.
}

\begin{abstract}
BACKGROUND: Assessing resident well-being is becoming increasingly important from a programmatic standpoint. Two measures that have been used to assess this are the Clance Impostor Scale (CIS) and the Maslach Burnout Inventory-Human Services Survey (MBI-HSS). However, little is known about the relationship between the two phenomena.
\end{abstract}

OBJECTIVES: To explore the prevalence and association between impostorism and burnout syndrome in a sample of internal medicine residents.

DESIGN: Anonymous, cross-sectional postal survey.

PARTICIPANTS: Forty-eight internal medicine residents (postgraduate year [PGY] 1-3) at the Schulich School of Medicine \& Dentistry (62.3\% response rate).

MEASUREMENTS AND MAIN RESULTS: Short demographic questionnaire, CIS and MBI-HSS. Impostorism and burnout syndrome were identified in $43.8 \%$ and $12.5 \%$ of residents, respectively. With the exception of a negative correlation between CIS scores and the MBI's personal accomplishment subscale $(r=-.30$; 95\% CI -.54 to -.02 ), no other significant relations were identified. Foreign-trained residents were more likely to score as impostors (odds ratio [OR] 10.7; 95\% CI 1.2 to 98.2) while senior residents were more likely to experience burnout syndrome (OR 16.5 95\% CI 1.6 to 168.5).

CONCLUSIONS: Both impostorism and burnout syndrome appear to be threats to resident well-being in our program. The lack of relationship between the two would suggest that programs and researchers wishing to address the issue of resident distress should consider using both measures. The finding that foreign-trained residents appear to be more susceptible to impostorism warrants further study.

KEY WORDS: impostorism; burnout syndrome; burnout;

resident well-being.

J Gen Intern Med 23(7):1090-4

DOI: $10.1007 / \mathrm{s} 11606-008-0536-\mathrm{x}$

(c) Society of General Internal Medicine 2008

\section{INTRODUCTION}

Residency is well-known to be a stressful period in medical training. In recent years, programmatic reforms, including duty-hour restrictions, have been made by individual programs and accrediting bodies to reduce resident stress. ${ }^{1,2}$ However, there continues to be a need to further explore issues related to stress and well-being, including the identification of appropriate measurement tools. Two possibly related phenomena that have been shown to affect resident well-being are impostorism and burnout syndrome.

Impostorism, characterized by chronic feelings of self-doubt and fear of being discovered as an intellectual fraud, was first described by Clance and Imes in a sample of college-educated females. ${ }^{3}$ Despite concrete evidence of their abilities, impostors were unable to internalize a sense of competence or skill ${ }^{4,5}$. Clance later identified feelings of impostorism in both sexes and across other highly successful populations; these feelings were especially intense at the beginning of new careers, jobs, or projects. ${ }^{6}$

Although the impostor phenomenon has been identified in students, teachers, and business professionals, ${ }^{7-9}$ it has not been well studied in medicine. Henning et al. surveyed a sample of medical, dental, nursing, and pharmacy students and found that $30 \%$ scored as impostors. ${ }^{10}$ In their sample, impostorism was found to be the strongest predictor of general psychological distress. Oriel et al. found that impostorism was prevalent in approximately one third of a sample of family medicine residents. ${ }^{11}$ While all of the respondents indicated that they were receiving the training necessary for a successful career, impostors suffered more psychological distress and did not believe they would be ready to practice family medicine after graduation.

In contrast to impostorism, the problem of burnout within the medical profession has received greater attention. Burnout, which has been defined as a three-dimensional syndrome involving occupationally-related feelings of emotional exhaustion, depersonalization, and professional inefficacy ${ }^{12,13}$ has been estimated to be prevalent in $10-76 \%$ of residents. ${ }^{2,14-17}$

In addition to studies of burnout syndrome, there is a growing body of literature addressing related elements. In a survey of residents from a variety of different training programs, over one third reported experiencing high levels of emotional strain ${ }^{18}$. Moreover, it has been suggested that surgical residents suffer greater degrees of emotional exhaustion than do their attending physicians. ${ }^{19}$ Medical trainees are 
also at risk of decreased humanism and empathy and increased cynicism during medical training. ${ }^{18,20-22}$

Although both impostorism and burnout have been shown to impact resident well-being and levels of psychological distress, to date, there have been no studies examining the relationship between the two. Specifically, there have been no studies exploring whether or not these two phenomena are independent. This is especially important if a program is trying to identify which aspects of resident well-being to assess. The purpose of the present study was to explore the prevalence and association between impostorism and burnout syndrome in a sample of internal medicine residents.

\section{METHODS}

\section{Study Design and Instruments}

From August to October 2006, all 77 residents in the 3-year internal medicine program at the Schulich School of Medicine \& Dentistry (University of Western Ontario, London, Canada) were invited to participate in an anonymous, cross-sectional postal survey. Survey mailings were managed by the Department of Medicine's Education Office with three follow-up mailings being performed at 2-week intervals. The study's protocol was reviewed and approved by the University of Western Ontario's Ethics Committee for Research Involving Human Subjects.

Short Demographic Questionnaire. Residents were asked to provide their gender, age category, marital status, program year, along with their residency status type (Canadian [CaRMS-matched] or foreign medical graduate).

Clance Impostor Scale. The Clance Impostor Scale (CIS) is a 20-item, cross-culturally validated instrument probing impostor-like feelings (e.g., I can give the impression that I am more competent than I really am). ${ }^{11,23}$ Items are scored along a five-point scale reflecting the level of agreement with the statement. Total scores can range from 20 to 100, with 62 or higher being indicative of impostorism. ${ }^{5,10}$

Maslach Burnout Inventory (Human Services Survey). The Maslach Burnout Inventory (Human Services Survey; MBI-HSS) is a 22-item questionnaire, which evaluates the frequency with which human service employees experience feelings or attitudes across three domains: 1) emotional exhaustion-nine items (e.g., "I feel emotionally drained from my work"); 2) depersonalizationfive items (e.g., "I don't really care what happens to some recipients"); and 3) personal accomplishment—eight items (e.g., "I feel I'm positively influencing other people's lives through my work"). ${ }^{24}$ All items are scored from 0 to 6 (never to every day). Using the norms for medical professionals, burnout prevalence was calculated according to the original Maslach high-degree definition of burnout syndrome: the combination of high emotional exhaustion ( $>=27$ points), high depersonalization ( $>=$ 10 points), and low personal accomplishment ( $<=33$ points). ${ }^{24}$

\section{Statistical Analyses}

All statistical calculations were performed using SPSS software (SPSS Inc [v12.0], Chicago, IL, USA). Participant demographics were described by frequency and percent response. Raw scores on the CIS and MBI-HSS were described with mean (SD) and minimum and maximum values while categorized scores were summarized in terms of their frequency (percent) of occurrence. Group differences were explored by means of one-way analysis of variance, independent $t$ test or test of independent proportions. Associations between categorized variables were explored by means of Fisher's Exact or chi-square tests of association and relative odds. Bivariate relationships between raw instrument scores were estimated with the use of Pearson Product Moment correlations ( $r$ ). Ninety-five percent confidence intervals were computed around group estimates, correlations, and relative odds.

\section{RESULT}

\section{Participant Demographics}

Surveys were returned by 48 residents (62.3\%). Approximately half were female, under 30 years old and single (Table 1). Responses were received from trainees across all training years and the return rates for these were proportionate to the overall program numbers. The majority of respondents were Canadian (CaRMS-matched) residents. There was a non-significant trend toward foreign medical graduates being less well-represented in our sample than in the overall program (14.6\% vs $28.6 \%$, $z=-1.8 ; p=.07)$.

\section{Impostorism}

The mean raw score for CIS responses was 61.2 (14.2 SD) (minimum 36, maximum 96; 95\% CI 57 to 65.2). Females $\left(t_{45}=-2.2, p=.03\right)$ and foreign medical graduates $\left(t_{7}=2.8\right.$, $p=.03$ ) reported significantly higher CIS scores (Table 2). Impostorism was identified in $43.8 \%$ of residents. Residency type (CaRMS-matched vs foreign medical graduates) was the only demographic variable found to be associated with impos-

Table 1. Participant and Program Demographics

\begin{tabular}{|c|c|c|}
\hline & $\begin{array}{l}\text { Respondents } \\
N=48(\%)\end{array}$ & $\begin{array}{l}\text { Total Program } \\
N=77(\%)\end{array}$ \\
\hline \multicolumn{3}{|l|}{ Gender } \\
\hline Female & $25(52.1)$ & 40 (51.9) \\
\hline Not Reported & $1(2.1)$ & \\
\hline \multicolumn{3}{|l|}{ Residency status type } \\
\hline Canadian (CaRMS-matched) & $39(81.2)$ & $55(71.4)$ \\
\hline Foreign medical graduate & 7 (14.6) & $22(28.6)$ \\
\hline Not Reported & $2(4.2)$ & \\
\hline \multicolumn{3}{|l|}{ Year of residency } \\
\hline One & $17(35.4)$ & $25(32.5)$ \\
\hline Two & $17(35.4)$ & $27(35)$ \\
\hline Three & $12(25)$ & $25(32.5)$ \\
\hline Not Reported & $2(4.2)$ & \\
\hline \multicolumn{3}{|l|}{ Age category } \\
\hline $20-29$ years & $40(83.3)$ & - \\
\hline 30-39 years & $7(14.6)$ & - \\
\hline Not Reported & $1(2.1)$ & - \\
\hline \multicolumn{3}{|l|}{ Marital status } \\
\hline Single & $31(64.6)$ & - \\
\hline Not Reported & $1(2.1)$ & - \\
\hline
\end{tabular}


Table 2. Resident Scores on the Clance Impostor Scale $(N=48)$

\begin{tabular}{|c|c|c|c|c|}
\hline & \multirow[t]{2}{*}{ Mean (SD) } & \multirow[t]{2}{*}{$95 \% \mathrm{Cl}^{*}$} & \multicolumn{2}{|l|}{ Impostors } \\
\hline & & & $n(\%)$ & $95 \% \mathrm{Cl}^{\dagger}$ \\
\hline \multicolumn{5}{|l|}{ Gender } \\
\hline Male & $56.4(11.5)$ & (51.3 to 61.4$)$ & $7(31.8)$ & (0.16 to 0.53$)$ \\
\hline Female & $65.2(15.5)^{\mathrm{T}}$ & (58.8 to 71.6 ) & $13(52)$ & (0.34 to 0.70$)$ \\
\hline \multicolumn{5}{|l|}{ Year of residency } \\
\hline One & $64(10)$ & (59.7 to 70$)$ & $10(58.8)$ & (0.36 to 0.78$)$ \\
\hline Two & $60.5(17.8)$ & (51.4 to 69.7 ) & $7(41.2)$ & (0.22 to 0.64$)$ \\
\hline Three & $58.3(12.9)$ & (50.1 to 66.5$)$ & $3(25)$ & (0.09 to 0.53$)$ \\
\hline \multicolumn{5}{|l|}{ Residency status type } \\
\hline Canadian (CaRMS-matched) & $58.3(11.8)$ & (54.5 to 62.1$)$ & $14(35.9)$ & (0.23 to 0.52$)$ \\
\hline Foreign medical graduate & $77.8(17.7)^{\mathrm{T}}$ & $(61.2$ to 94$)$ & $6(85.7)$ & (0.49 to 0.97$)$ \\
\hline \multicolumn{5}{|l|}{ Impostorism } \\
\hline Yes & & & $21(43.8)$ & (0.31 to 0.58$)$ \\
\hline No & & & 27 (56.3) & (0.42 to 0.69$)$ \\
\hline
\end{tabular}

*95\% CI for mean

† $95 \%$ CI for proportion

${ }^{\mathrm{F}}$ Significantly different at $p=.03$

torism (Fisher's Exact test, $p=.01$ ); all but one foreign medical graduate scored as an impostor (85.7\%) compared to $35.9 \%$ of Canadians (OR=10.7; 95\% CI 1.2 to 98.2).

\section{Burnout Syndrome}

The mean raw score for residents on the emotional exhaustion subscale was 22.5 (9.8 SD) (minimum 5, maximum 44; 95\% CI 19.6 to 23.5), with nearly a third reporting high degrees of emotional exhaustion (Table 3). No associations were detected between residents' emotional exhaustion and the demographic variables.

The mean raw score for depersonalization was 10.1 (6 SD) (minimum 1, maximum 28; 95\% CI 8.4 to 11.9 ), with $54.2 \%$ of residents reporting highly negative and detached attitudes (Table 3). More than half of the residents reported regularly (a few times a month or more) feeling that they have become more callous toward people since entering the program. Almost $40 \%$ reported worrying regularly that their residency was "hardening them emotionally" and perceived that they were treating

Table 3. Resident Scores on the Maslach Burnout Inventory (Human Services Survey)

\begin{tabular}{lrr}
\hline \hline & $n(\%)$ & $95 \% \mathrm{Cl}^{*}$ \\
\hline $\begin{array}{l}\text { Emotional Exhaustion } \\
\text { Low }(<=18)\end{array}$ & $19(39.6)$ & \\
Moderate (19-26) & $15(31.3)$ & $(0.27$ to 0.54$)$ \\
High $(>=27)$ & $14(29.2)$ & $(0.20$ to 0.45$)$ \\
Depersonalization & $13(27.1)$ & $(0.18$ to 0.43$)$ \\
Low $(<=5)$ & $9(18.8)$ & $(0.17$ to 0.41$)$ \\
Moderate (6-9) & $26(54.2)$ & $(0.10$ to 0.32$)$ \\
High $(>=10)$ & $13(27.1)$ & $(0.40$ to 0.67$)$ \\
Personal Accomplishment & $20(41.7)$ & $(0.17$ to 0.41$)$ \\
Low $(<=33)$ & $15(31.3)$ & $(0.29$ to 0.56$)$ \\
Moderate (34 -39$)$ & $6(12.5)$ & $(0.20$ to 0.45$)$ \\
High $(>=40)$ & $42(87.5)$ & $(0.06$ to 0.25$)$ \\
Burnout Syndrome & & $(0.75$ to 0.94$)$ \\
Yes & & \\
No & & \\
\hline
\end{tabular}

"95\% CI for the proportion some of the recipients of their care as "impersonal objects" at least a few times per month. Moreover, nine (19\%) residents reported that they regularly "don't really care what happened to some recipients" of their care. No associations were detected between depersonalization and the demographic variables.

The mean raw score for personal accomplishment was 36.4 (6.4 SD) (minimum 22, maximum 48; 95\% CI 34.5 to 38.2 ). Less than a third scored within the low range on this subscale. Overall, $79.2 \%$ indicated that at least once a week they felt that they "had accomplished many worthwhile things in this job." Impostors did not differ from non-impostors on this question (Fisher's Exact test, $p=.08$ ).

The predominant patterns for scores on the MBI-HSS were high levels of depersonalization, a moderate to high sense of personal accomplishment combined with varying degrees of emotional exhaustion. In terms of individual subscale prevalence, high levels of depersonalization were more common than emotional exhaustion.

Six residents (12.5\%) met the criteria for burnout syndrome (Table 3). A significant association was detected between residency year and burnout syndrome $\left(x_{2}^{2}=8.8, p=.01\right)$. All but one of the residents who met the criteria for burnout syndrome and who revealed their year of training were senior residents (80\%; OR $16.595 \%$ CI 1.6 to 168.5). No other associations were detected between burnout syndrome and the demographic variables.

Inter-correlations between the individual subscales revealed a significant positive relationship between emotional exhaustion and depersonalization $(\mathrm{r}=.53, p=.00,95 \% \mathrm{CI} .29$ to 71$)$ and a significant negative correlation between emotional exhaustion and personal accomplishment $(r=-.30, p=.04$, $95 \%$ CI -0.54 to -0.02 ).

\section{Association between Impostorism and Burnout Syndrome}

Three out of the six residents who met the criteria for burnout also scored as impostors. A significant negative correlation was detected between raw scores on the personal accomplishment subscale and the CIS ( $r=-.30, p=.04 ; 95 \%$ CI -0.54 to -0.02$)$. No other significant relationships were found. 


\section{DISCUSSION}

The results of our study illustrate several areas of concern with regard to resident well-being. Starting with the programmatic issues, we are worried about the number of residents reporting a considerable degree of self-doubt and/or high degrees of workplace-related distress, especially those six residents suffering from burnout syndrome.

Our resident population demonstrated a prevalence of impostorism similar to that found in other health professions trainees. ${ }^{10,11}$ Further, female residents had higher scores on the impostor scale, a finding that is also consistent with the existing literature. ${ }^{7,10,11}$ As in the Oriel study, there was no association detected between program year and feelings of impostorism. ${ }^{11}$ This is somewhat disconcerting as educators would hope to see feelings of inadequacy or self-doubt decrease with training and experience. While this may seem to suggest that impostorism represents a stable trait, residency is a time where trainees are repeatedly being asked to adapt to new clinical rotations, with different expectations, responsibilities, and pressures. Therefore, this result is consistent with Clance's suggestion that impostor feelings are pronounced when one is faced with new challenges. ${ }^{6}$ Future longitudinal research is required to examine potential changes in these beliefs over time.

It is interesting to note that a significant association was detected between the prevalence of impostorism and residency status type. All but one non-Canadian resident was classified as an impostor (85.7\%) compared to approximately $36 \%$ of CaRMS-matched residents. To our knowledge, the present study is one of the few existing papers to report that international medical residents appear to worry more that they are intellectually inadequate and perceive themselves to be less competent than colleagues who are training within their country of citizenship. Given that most international medical graduates come from non-English speaking countries, ${ }^{25}$ we should not be too surprised to find out that they appear to be vulnerable to these feelings as they are faced with the expectations and pressures of a residency program that practices in a language that is not their native tongue. Fiscella et al. explored previously unacknowledged feelings in a sample of international medical graduates and found that fears of rejection and disappointing patients due to perceived language barriers emerged as prevalent issues for these residents. ${ }^{26}$ Given that foreign graduates account for up to $20 \%$ of North American postgraduate medical trainees, this finding, if replicated, could have significant implications for the development of programs addressing the issue of resident well-being. ${ }^{27}$

Burnout syndrome was detected in six residents. All but one of these individuals was in their third year of training. In comparing our results with those from other programs, they at first appear to be among the lowest reported in the literature. However, upon further inspection, this may not be the case. Rather than calculating prevalence in terms of all three of the Maslach dimensions, some researchers have based their estimates upon less stringent criteria. Shanafelt et al., who based their estimates on the presence of a single high-scoring dimension (either high emotional exhaustion or high depersonalization) detected a burnout prevalence of $76 \% .{ }^{14}$ When we recalculated our scores based on their methodology we too found a much higher prevalence (56.3\%), suggesting that our findings are more similar than they would have at first appeared. Moreover, we believe, that to use such a liberal definition would make the problem appear more prevalent than it really is. As Maslach has said "to limit the concept of burnout to just the component of emotional exhaustion is to define it simply as experienced stress and nothing more". ${ }^{12}$ This issue has also been recently commented on by Prins et al. who indicated that using a single Maslach dimension increases the probability of false positives. ${ }^{28}$

Close to $30 \%$ of residents scored within the high range for emotional exhaustion. Given that Maslach has said that emotional exhaustion most closely approximates an orthodox stress variable, it appears then that a substantial proportion of trainees are experiencing a high degree of work-related stress. ${ }^{12}$ Perhaps of even greater importance is the finding that more than half of the respondents admitted to experiencing highly negative and detached attitudes toward patients (scored in the high range for depersonalization), with almost $40 \%$ indicating that they viewed patients as "impersonal objects" at least a few times a month and nearly one in five reporting that they regularly "don't really care" what happens to some patients. It has been theorized that emotional exhaustion is the first stage in the development of burnout syndrome and that depersonalized attitudes emerge subsequently as an attempted coping strategy. ${ }^{29}$ Although raw scores on the emotional exhaustion and depersonalization subscales were found to be strongly correlated, high levels of depersonalization were found to be more prevalent than emotional exhaustion. This suggests that a number of residents are experiencing depersonalized attitudes that may not be attributable to workplace stress. ${ }^{30}$ This possibility is supported by other studies showing decreased humanism and empathy and increased cynicism during medical training. ${ }^{18,20-22}$

To our knowledge, the present study was the first to explore the relation between impostorism and burnout syndrome. Although we had anticipated a strong correlation between the two, only three residents presented scores indicative of both impostorism and burnout syndrome. This result may have been influenced by the sample size. However, the weak negative correlation between CIS and the personal accomplishment subscale suggests that at best, impostor feelings may impair one's ability to develop a strong sense of accomplishment. Personal accomplishment scores buffered a number of residents from being classified with burnout syndrome including six resident impostors. Impostors were found to be no less likely to report feeling satisfied on a regular basis with their achievements in the program. Therefore, it appears that despite their insecurities, they still perceive themselves to be able to function effectively. How we can assist them to do so more comfortably remains to be explored.

It also needs to be highlighted that half of the residents who participated in this study did not present scores that were indicative of impostorism or burnout syndrome. As much as it is important to focus future research on identifying factors that contribute to residents' distress, it is equally important that attention be directed toward uncovering characteristics that help physicians to be resilient to such effects.

This study does have some methodological limitations. The most important of these is the overall low prevalence of burnout syndrome in the sample which may have limited our ability to detect a relationship between burnout syndrome and impostorism. That being said, as mentioned above, if a relationship does exist it is unlikely to be strong. Other 
limitations include the fact that this was a single-institution study and that our response rates were somewhat low, especially for foreign graduates. With regard to generalizability, the demographics of our residents and the requirements of training are likely to be very similar to other North American programs. Moreover, arguments against self-selection and social desirability biases playing a major role in this study include the anonymous nature of the survey, the apparent demographic representativeness of respondents and the fact that stress, burnout, impostorism, and resident well-being had not been discussed in any formal manner by our program prior to the study. Prevalence estimates reported by our study are similar to those presented by other authors in the area. In addition, it has been previously demonstrated that the MBI is resistant to the threat of social desirability bias. ${ }^{24}$

The results of this study very much support the suggestion that more work is needed to address the issue of resident wellbeing. In our study, a large number of residents were found to suffer from some form of distress. However, the CIS and MBI-HSS appeared to identify residents with distinct forms of this. Given the large numbers of residents with impostorism or high levels of depersonalization in the absence of emotional exhaustion, interventions focusing solely on reducing workplace stress may not be enough. Moreover, both measurement tools should be used by those attempting to measure resident well-being.

Acknowledgments: The authors express gratitude to those who participated in this study. We also would like to thank the Department of Medicine's Education Office for managing the study's mailings.

Conflict of Interest: The authors declare no conflict of interest with regards to this study.

Corresponding Author: Mark A. Goldszmidt, MD, MHPE, FRCPC, Research Park (MOG), Suite 227, 100 Collip Circle, London, ON, Canada N6G 4X8 (e-mail: mgoldszm@uwo.ca).

\section{REFERENCES}

1. Accreditation Council of Graduate Medical Education. Resident duty hours. http://www.acgme.org/DutyHours/dutyHrs_Index.asp.

2. Gopal R, Glasheen JJ, Miyoshi TJ, Prochazka AV. Burnout and internal medicine resident work-hour restrictions. Arch Intern Med. 2005; 165(22):2595-600.

3. Clance P, Imes SA. The impostor phenomenon in high achieving women: dynamics and therapeutic intervention. Psychotherapy (Chic Ill). 1978;15(3):241-7.

4. Langford $\mathbf{J}$, Clance $\mathbf{P}$. The impostor phenomenon: recent research findings regarding dynamics, personality and family patterns and their implications for treatment. Psychotherapy. 1993;30:495-501.

5. September AN, McCarrey M, Baranowsky A, Parent C, Schindler D. The relation between well-being, impostor feelings, and gender role orientation among Canadian university students. J Soc Psychol. 2001; 141(2):218-32.

6. Clance PR. The Impostor Phenomenon: When Success Makes You Feel Like a Fake. New York: Bantam Books.
7. King JE, Cooley EL. Achievement orientation and the impostor phenomenon among college students. Contemp Educ Psychol. 1995;20 (3):304-12.

8. Sonnak C, Towell T. The impostor phenomenon in British university students: relationships between self-esteem, mental health, parental rearing style and socioeconomic status. Pers Individ Differ. 2001;31 (6):863-74.

9. Sightler KW, Wilson MG. Correlates of the impostor phenomenon among undergraduate entrepreneurs. Psychol Rep. 2001;88(3):679-89.

10. Henning $\mathbf{K}$, Ey $\mathbf{S}$, Shaw D. Perfectionism, the imposter phenomenon and psychological adjustment in medical, dental, nursing and pharmacy students. Med Educ. 1998;32(5):456-64.

11. Oriel K, Plane MB, Mundt M. Family medicine residents and the impostor phenomenon. Fam Med. 2004;36(4):248-52.

12. Maslach C. Burnout: a Multidimensional Perspective. In: Schaufeli WB Maslach C, Marek T, eds. Professional Burnout: Recent Developments in Theory and Research. Washington DC: Taylor \& Francis; 1993:19-32.

13. Maslach C, Schaufeli WB, Leiter MP. Job burnout. Annu Rev Psychol. 2001;52:397-422

14. Shanafelt TD, Bradley KA, Wipf JE, Back AL. Burnout and selfreported patient care in an internal medicine residency program. Ann Intern Med. 2002;136(5):358-67.

15. Panagopoulou E, Montgomery A, Benos A. Burnout in internal medicine physicians: differences between residents and specialists. Eur J Intern Med. 2006;17(3): 195-200.

16. Martini S, Arfken CL, Churchill A, Balon R. Burnout comparison among residents in different medical specialties. Acad Psychiatry. 2004; 28(3):240-2.

17. Golub JS, Weiss PS, Ramesh AK, Ossoff RH, Johns MM 3rd. Burnout in residents of otolaryngology-head and neck surgery: a national inquiry into the health of residency training. Acad Med. 2007;82(6):596-601.

18. Biaggi P, Peter S, Ulich E. Stressors, emotional exhaustion and aversion to patients in residents and chief residents-what can be done? Swiss Med Wkly. 2003;133(23-24):339-46.

19. Sargent MC, Sotile W, Sotile MO, Rubash H, Barrack RL. Stress and coping among orthopaedic surgery residents and faculty. J Bone Joint Surg Am. 2004;86-A(7):1579-86.

20. Collier VU, McCue JD, Markus A, Smith L. Stress in medical residency: status quo after a decade of reform? Ann Intern Med. 2002;136(5): 384-90.

21. Bellini LM, Baime M, Shea JA. Variation of mood and empathy during internship. JAMA. 2002;287(23):3143-6.

22. Eron LD. Effect of medical education on medical students' attitudes. J Med Educ. 1955;30:559-66.

23. Chae J, Piedmont R, Estadt B, Wicks R. Personological evaluation of Clance's impostor phenomenon scale in Korean sample. J Pers Assess. 1995;65(3):486

24. Maslach C, Jackson SE, Leiter MP. Maslach Burnout Inventory Manual. 3Mountain View, California: CPP; 1996.

25. Kuczkowski KM. International medical school graduates in American medicine: is there the "dark side of the moon"? Acta Obstet Gynecol Scand. 2004;83(12):1228-9.

26. Fiscella K, Roman-Diaz M, Lue BH, Botelho R, Frankel R. "Being a foreigner, I may be punished if I make a small mistake": Assessing transcultural experiences in caring for patients. Fam Pract. 1997; 14(2):112-6

27. Bates J, Andrew R. Untangling the roots of some IMG's poor academic performance. Acad Med. 2001;76(1):43-6.

28. Prins JT, Gazendam-Donofrio SM, Tubben BJ, van der Heijden FM, van de Wiel HB, Hoekstra-Weebers JE. Burnout in medical residents: a review. Med Educ. 2007;41(8):788-800.

29. Leiter MP, Maslach C. The impact of interpersonal environment on burnout and organizational commitment. J Org Behav. 1988;9(4):297308.

30. Leiter MP. Coping patterns as predictors of burnout: the function of control and escapist coping patterns. J Org Behav. 1991;12(2):123-144. 